\title{
Peiminine Inhibits Glioblastoma in Vitro and in Vivo Through Cell Cycle Arrest and Autophagic Flux Blocking
}

\author{
Boxian Zhao $\mathrm{O}^{\mathrm{a}, \mathrm{b}, \mathrm{c}} \quad$ Chen Shen ${ }^{\mathrm{a}, \mathrm{b}, \mathrm{c}} \quad$ Zhixing Zheng ${ }^{\mathrm{a}, \mathrm{b}, \mathrm{c}} \quad$ Xiaoxiong Wang $^{\mathrm{a}, \mathrm{b}, \mathrm{c}}$ \\ Wenyang Zhao ${ }^{a, b, c} \quad$ Xin Chen ${ }^{a, b, c} \quad$ Fei Peng ${ }^{a, b, c}$ Linmeng Xue $e^{a, b, c}$ \\ Mengting Shu $u^{a, b, c} \quad X$ Hou $^{a, b, c} \quad$ Kaikai Wang ${ }^{a, b, c} \quad$ Chen Zhong ${ }^{a, b, c}$ \\ Jingxian Sun ${ }^{a, b, c}$ Jinzhao Wan ${ }^{a, b, c}$ Shiguang Zhao ${ }^{a, b, c}$ \\ aDepartment of Neurosurgery, The First Affiliated Hospital of Harbin Medical University, Harbin, \\ bInstitute of Brain Science, Harbin Medical University, Harbin, Institute of Neuroscience, Sino-Russian \\ Medical Research Centre, Harbin Medical University, Harbin, China
}

\section{Key Words}

Peiminine $\cdot \mathrm{GBM} \cdot$ Cell cycle • Autophagy $•$ AKT-GSK3 $\beta$ - AMPK-ULK1

\begin{abstract}
Background/Aims: Glioblastoma multiforme (GBM) is the most devastating and widespread primary central nervous system tumour in adults, with poor survival rate and high mortality rates. Existing treatments do not provide substantial benefits to patients; therefore, novel treatment strategies are required. Peiminine, a natural bioactive compound extracted from the traditional Chinese medicine Fritillaria thunbergii, has many pharmacological effects, especially anticancer activities. However, its anticancer effects on GBM and the underlying mechanisms have not been demonstrated. This study was conducted to investigate the potential antitumour effects of peiminine in human GBM cells and to explore the related molecular signalling mechanisms in vitro and in vivo. Methods: Cell viability and proliferation were detected with MTT and colony formation assays. Morphological changes associated with autophagy were assessed by transmission electron microscopy (TEM). The cell cycle rate was measured by flow cytometry. To detect changes in related genes and signalling pathways in vitro and in vivo, RNA-seq, Western blotting and immunohistochemical analyses were employed. Results: Peiminine significantly inhibited the proliferation and colony formation of GBM cells and resulted in changes in many tumour-related genes and transcriptional products. The potential anti-GBM role of peiminine might involve cell cycle arrest and autophagic flux blocking via changes in expression of the cyclin D1/CDK network, p62 and LC3. Changes in flow cytometry results and TEM findings were also observed. Molecular alterations included downregulation of the expression of not only phospho-Akt and phospho-GSK3 $\beta$ but also phospho-AMPK and phospho-ULK1. Furthermore, overexpression of AKT and inhibition of AKT reversed and augmented peiminine-induced cell cycle arrest in GBM cells, respectively. The

B. Zhao, C. Shen and Z. Zheng contributed equally to this work.

\begin{tabular}{ll}
\hline Shiguang Zhao & Department of Neurosurgery, The First Affiliated Hospital of Harbin Medical University \\
& No.23 Youzheng Street, Nangang District, Harbin 150001, Heilongjiang Province (China) \\
& Tel. 86-451-85552799, Fax 86-451-53629254, E-Mail guangsz@hotmail.com
\end{tabular}
\end{abstract}




\section{Cellular Physiology Cell Physiol Biochem 2018;51:1566-1583

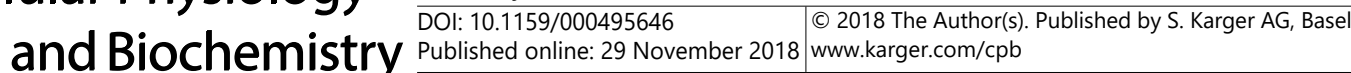 \\ Zhao et al.: Antitumour Effect of Peiminine on Glioblastoma}

cellular activation of AMPK reversed the changes in the levels of protein markers of autophagic flux. These results demonstrated that peiminine mediates cell cycle arrest by suppressing AktGSk3 $\beta$ signalling and blocks autophagic flux by depressing AMPK-ULK1 signalling in GBM cells. Finally, peiminine inhibited the growth of U251 gliomas in vivo. Conclusion: Peiminine inhibits glioblastoma in vitro and in vivo via arresting the cell cycle and blocking autophagic flux, suggesting new avenues for GBM therapy.

(C) 2018 The Author(s)

Published by S. Karger AG, Basel

\section{Introduction}

Glioblastoma multiforme (GBM) is a grade IV glioma according to the World Health Organization (WHO) classification and is the most widespread primary central nervous system tumour in adults [1]. In past decades, even GBM patients who were treated via surgery, radiotherapy, chemotherapy, etc. showed a median survival of less than 15 months, with a 5-year relative survival of less than $3 \%$ and a mortality rate of nearly $100 \%[2,3]$. The existing treatments cannot bring greater benefits to patients, and more novel treatment strategies are required [4]. In recent years, increasing numbers of traditional Chinese medicine monomers have shown extraordinary therapeutic effects on cancer [5, 6]. A new weapon against GBM might be found in these natural medicine monomers.

Fritillaria thunbergii (Chinese name Zhe Beimu), mainly distributed in Jiangsu Province and Zhejiang Province in China and in a few areas in Japan, has been used to treat furuncle and lung diseases in traditional Chinese medicine for thousands of years. Pathological and biological studies have confirmed that the active principle compounds of Fritillaria thunbergii are alkaloids, including peimisine, peimine and peiminine $[7,8]$. In recent studies, peiminine has been reported to have anti-inflammatory, analgesic, and antitussive effects and to be able to ameliorate acute lung injury [9-12]. In addition, it also has the potential to fight cancer [13]. However, the antitumour effects of peiminine against GBM have not been reported.

It has been reported that peiminine represses colorectal carcinoma cell proliferation and induces autophagic cell death [13]. As is well known, the protein kinase B (AKT)-glycogen synthase kinase $3 \beta$ (GSK3 $\beta$ ) pathway controls many intracellular processes involved in cancer cell growth, proliferation, angiogenesis, metabolism and motility in GBM [14, 15]. Many studies have shown that inhibiting the AKT pathway and its downstream effector, GSK3 $\beta$, reduced the growth of GBM cells [16, 17]. AMP-activated protein kinase (AMPK), as a serine/ threonine kinase that negatively regulates lipid anabolism, is activated by metabolic stress to promote the process of catabolism in general. AMPK has also been shown to be a positive regulator of autophagy [18]. Extensive evidence confirms that AMPK promotes the positive regulation of ULK1, an important autophagic factor, through phosphorylation $[18,19]$. In contrast, dampened Akt signalling enhances autophagy by increasing ULK1 phosphorylation [20]. Although ULK1 is regulated by the AKT and AMPK signalling pathways, inhibition of AMPK signalling can lead to autophagy reversal by suppressing the Akt-mTOR signalling pathway [21].

Most interestingly, we determined that the mechanism of the antitumour effect of peiminine on GBM is different from that described in other reports regarding its role in colon tumours. In this study, we show the efficacious inhibitory effect of peiminine on GBM occurs through the AKT-GSK3 $\beta$ and AMPK-ULK1 pathways. However, we found a difference from other studies in that peiminine does not mediate the autophagy of GBM cells but rather blocks the autophagic flux. Moreover, peiminine also causes cell cycle arrest at the G1/G0 stage. Our findings suggest not only the potential use of peiminine for the treatment of GBM but also different mechanisms of action for peiminine in different tumours, which may provide new avenues for cancer therapy. 


\section{Cellular Physiology Cell Physiol Biochem 2018;51:1566-1583

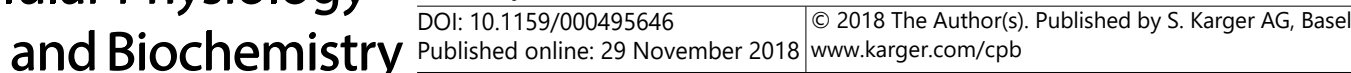 \\ Zhao et al.: Antitumour Effect of Peiminine on Glioblastoma}

\section{Materials and Methods}

\section{Cell culture}

The human GBM cell lines used in our study were purchased from the RIKEN Cell Bank (Tsukuba Science City, Japan). The GBM cell lines were cultured using Dulbecco's Modified Eagle's Medium (DMEM; Corning, 10-017-CVR) with $10 \%$ foetal bovine serum (FBS; Invitrogen), and $1 \%$ penicillin and streptomycin (Sigma-Aldrich, USA) at $37^{\circ} \mathrm{C}$ with $5 \% \mathrm{CO}_{2}$ in an incubator.

\section{Reagents}

Peiminine (Aladdin, CAS18059-10-4) was prepared as a solution in dimethyl sulfoxide (DMSO) and diluted to the indicated concentrations. LY294002 (MCE, HY-10108) and metformin hydrochloride (MCE, HY-17471A) were purchased from MedChem Express (New Jersey, USA). The primary antibodies used in this study recognized the following proteins: $\beta$-actin (ABclonal Technology, AC004), p62/SQSTM1 (SigmaAldrich, P0067), LC3 (Sigma-Aldrich, L7543), p-AKT Ser473 (Cell Signaling Technology, 9271), AKT (Cell Signaling Technology, 9272), PI3K (Cell Signaling Technology, 4257), p-PI3K p85 (Tyr458)/p55 (Tyr199) (Cell Signaling Technology, 4228), p-GSK3 $\alpha / \beta$ Ser21/9 (Cell Signaling Technology, 8566), GSK3 $\alpha / \beta$ (Cell Signaling Technology, 5676), p-Rb Ser345 (Cell Signaling Technology, 9301), p-ULK1 Ser556 (Abcam, 133747), ULK1 (Abcam, 128859), p-AMPK Thr172 (Cell Signaling Technology, 2535), AMPK (Cell Signaling Technology, 2532), cyclin D1 (Abcam, 134175), p21 (Wanleibio, 362), p27 (Wanleibio, 1769), CDK2 (Abcam, 32147), and CDK4 (Wanleibio, 1711).

\section{Transcriptome shotgun sequencing (RNA-seq)}

Total RNA was extracted using TRIzol reagent (Invitrogen, USA) following the manufacturer's procedure. Quantity and purity of the total RNA were analysed via a 2100 Bioanalyzer instrument and RNA 6000 Nano Kit (Agilent, USA) with an RNA integrity number (RIN) > 7.0. Approximately $10 \mu$ g of total RNA representing a specific adipose type was subjected to isolation of poly(A)-mRNA with poly-T oligo-attached magnetic beads (Invitrogen). Following purification, the mRNA was fragmented into small pieces using divalent cations under an elevated temperature. Then, the cleaved RNA fragments were reverse-transcribed to create the final cDNA library in accordance with the protocol for the mRNA Seq Sample preparation kit (Illumina, San Diego, USA). The average insert size for the paired-end libraries was $300 \pm 50 \mathrm{bp}$. In addition, we performed paired-end sequencing on an Illumina X10 instrument (LC Sciences, USA) following the vendor's recommended protocol.

\section{MTT and clonogenic assays}

GBM cells were seeded at $5 \times 10^{4} / \mathrm{ml}$ with $200 \mu \mathrm{l}$ of culture medium on 96 -well plates and treated with the indicated concentration of peiminine. At 12,24 , and $48 \mathrm{~h}$ after treatment, $10 \mu \mathrm{l}$ of MTT dye $(5 \mathrm{mg} / \mathrm{ml}$, Cat\# M2128, Sigma, USA) was added to each well, and the plates were incubated at $37^{\circ} \mathrm{C}$ in an incubator for $4 \mathrm{~h}$. Afterwards, the supernatants were replaced by $150 \mu \mathrm{l}$ of DMSO (Cat\# D2650, Sigma, USA) to stop the reactions. The absorbance at $490 \mathrm{~nm}$ was measured using a spectrophotometer (Tecan, Switzerland). To determine the long-term effects, cells were seeded in six-well plates at a density of 150 cells/well and treated with peiminine at different concentrations $(0,100,200$ or $400 \mu \mathrm{M})$ for 7 days. Next, cells were rinsed with fresh medium every 3 days prior to staining with crystal violet $(0.4 \mathrm{~g} / \mathrm{L})$. The number of cells was counted using ImageJ software.

\section{Western blot analysis}

GBM cells were seeded onto 6-cm dishes and treated with peiminine at different concentrations and times. Then, the cells were collected with radioimmunoprecipitation assay (RIPA) lysis buffer (Thermo Scientific, USA) with a protease inhibitor (Roche, 05892970001) and a phosphatase inhibitor (Roche, 4906845001). According to the manufacturer's instructions, the protein density was quantified by a bicinchoninic acid (BCA) assay kit (Beyotime, P0010). Using 7.5-12.5\% SDS gels, lysates (15-100 $\mu \mathrm{g}$ ) were separated by SDS-PAGE and then transferred to PVDF membranes (Millipore, Bedford, MA, USA). The membranes were blocked in $5 \%$ skim milk with Tween at room temperature for $1 \mathrm{~h}$ and incubated with the indicated primary antibodies at $4{ }^{\circ} \mathrm{C}$ overnight. After washing with TBST, the membranes were incubated 


\section{Cellular Physiology Cell Physiol Biochem 2018;51:1566-1583 and Biochemistry \begin{tabular}{l|l}
$\bar{D}$ DOI: 10.1159/000495646 & $\begin{array}{l}\text { (c) } 2018 \text { The Author(s). Published by S. Karger AG, Basel } \\
\text { www.karger.com/cpb }\end{array}$
\end{tabular} \\ Published online: 29 November 2018 whis.karger.com/cpb}

with the secondary antibodies conjugated to horseradish peroxidase (ZSGB-BIO, ZF-2301, ZF-2305) for 1 $\mathrm{h}$ at room temperature. The protein bands were visualized using the Beyo ECL Star (Beyotime, P0018A) detection kit and quantified with ImageLab software.

\section{Cell cycle detected by flow cytometry}

LN229 and U251 cells at a density of $5 \times 10^{5}$ cells/well were exposed to peiminine at different concentrations $(0,100,200$ or $400 \mu \mathrm{M})$ for $24 \mathrm{~h}$. Then, cells were suspended in $70 \%$ ethanol and fixed at $4{ }^{\circ} \mathrm{C}$ for $12 \mathrm{~h}$. The treated cells were incubated with propidium iodide (PI) according to the protocol of the Cell Cycle and Apoptosis Analysis Kit (Beyotime, P0010). Then, the stained cells were analysed by an Accuri C6 flow cytometer (BD Bioscience), and the distribution of cells in different phases was calculated via BD Accuri C6 software.

\section{Transmission electron microscopy}

LN229 and U251 cells were seeded onto 6-cm dishes for full attachment. After peiminine $(200 \mu \mathrm{M})$ treatment for $24 \mathrm{~h}$, cells were harvested and fixed by $2.5 \%$ precooled glutaraldehyde at $4{ }^{\circ} \mathrm{C}$ overnight. Then, cells were dehydrated by ethanol and acetone and fixed with $1 \%$ osmium tetroxide for 30 min. Cells were then embedded in araldite, and ultrathin sections were obtained (50-60 nm). The sections were contrasted with lead citrate-uranyl acetate and examined with a TEM (Hitachi H-7650, Japan).

\section{Lentivirus transductions}

Lentiviral particles containing Lv-AKT and virus without AKT, which served as a negative control (Lvcontrol), were purchased from Wanleibio (Shenyang, China) and transduced into GBM cells following the manufacturer's instructions. The virus-infected cells were GFP-positive and were sorted out by an FSX100 Bio Imaging Navigator (Olympus, Japan) after $72 \mathrm{~h}$.

\section{Tumourigenicity in nude mice}

Animal experiments were approved by the Animal Care and Ethical Committee of the First Affiliated Hospital of Harbin Medical University and conducted based on the state guidelines from the Ministry of Science and Technology of China. Six-week-old female BALB/c nude mice were purchased from Vital River Laboratory Animal Technology Co. Ltd. (Beijing, China). U251 cells $\left(1 \times 10^{6}\right.$ cells in $100 \mu$ of PBS) were injected into the right hips of each mouse, and the mice were randomly assigned into one of the two groups $(n=6)$. One week post-implantation, the control group received intraperitoneal injections of saline every other day while the treatment group received intraperitoneal injections of $2 \mathrm{mg} / \mathrm{kg}$ peiminine every other day. At 21 days after tumour implantation, all mice were euthanized, tumours were isolated, and the tumour weight was recorded.

\section{Immunocytochemistry}

Tumour tissues were fixed by formalin and processed into paraffin-embedded samples. Then, the samples were sliced into 5 - $\mu$ m-thick sections. The tumour sections were incubated in $\mathrm{H}_{2} \mathrm{O}_{2}$ for 10 min and immunostained for the indicated primary antibodies at $4{ }^{\circ} \mathrm{C}$ overnight. A secondary antibody (ZSGB-BIO, PV6001) was applied for $30 \mathrm{~min}$ at $37^{\circ} \mathrm{C}$. The sections were developed with the Polink-2 Plus ${ }^{\circledR}$ Polymer HRP Detection System (ZSGB-BIO, PV-9001) following the manufacturer's instructions. In addition, the sections were visualized with a diaminobenzidine substrate kit (ZSGB-BIO, ZLI-9019). Then, tumour tissue sections were counterstained by haematoxylin, dehydrated and cover slipped. Finally, the sections were surveyed by a FSX100 Bio Imaging Navigator (Olympus, Japan). Data were analysed with Image-Pro Plus 6.0.

\section{Statistical analysis}

The data from three repeated independent experiments are expressed as the mean \pm SD. Comparisons between groups were performed using two-tailed Student's t-tests. A value of $\mathrm{p}<0.05$ was considered statistically significant. 


\section{Cellular Physiology Cell Physiol Biochem 2018;51:1566-1583

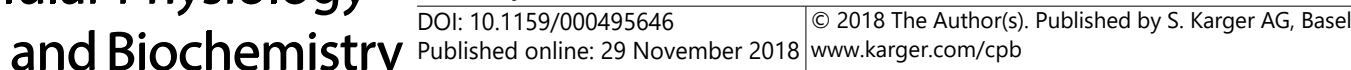

\section{Results}

Peiminine inhibits the proliferation and colony formation of GBM cells

First, we used an MTT assay to validate the effect of peiminine on GBM cell viability and calculate the inhibitory concentration ( $\mathrm{IC}_{50}$ Table 1). Fig. 1a shows that U251, LN229, U87 and T98 cell growth was inhibited with different concentrations of peiminine for $12 \mathrm{~h}$, $24 \mathrm{~h}$ and $48 \mathrm{~h}$. Moreover, we used U251 and LN229 cells for the colony forming assay because these cell lines have the largest and smallest IC50 values, respectively. Also, we used these cell lines in the subsequent experiments for the same reason. The U251 and LN229 cells were treated with peiminine at different concentrations $(0,100,200$ or $400 \mu \mathrm{M}$ ) and analysed by a colony formation assay (Fig. 1b and c). The results indicated that peiminine has a long residual ability to inhibit cell proliferation.

Altered expression of cancerassociated genes by peiminine in GBM cells

We used transcriptome shotgun sequencing (RNA-seq) to identify cancer-associated gene signatures in GBM cells treated with peiminine. As the LN229 cell line has the largest IC50, we selected the LN229 cells for the RNA-seq experiment. The differential expression of genes and transcripts in the LN229 cells treated with peiminine $(200 \mu \mathrm{M}$, $24 \mathrm{~h}$ culture) and the controls was clear (Fig. 2a and b). Peiminine treatment also resulted in increased or decreased expression of many genes involved in different aspects of cancer cell development, including proliferation, cell metabolism, cell cycle, autophagy, apoptosis, cell migration, angiogenesis, cell adhesion and the inflammatory response (Fig. 2c). The expression of many cancer-associated signalling pathways, such as the PI3K-AKT, AMPK, MAPK, p53 and Wnt pathways, was altered.
Table 1. Average half maximal inhibitory concentration for cell growth in GBM cell lines after exposure to peiminine for $12 \mathrm{~h}, 24 \mathrm{~h}$ and $96 \mathrm{~h}$

\begin{tabular}{lccc}
\hline & \multicolumn{3}{c}{ IC50 of (Peiminine $\mu \mathrm{M})$} \\
& $12 \mathrm{~h}$ & $24 \mathrm{~h}$ & $36 \mathrm{~h}$ \\
\hline U87 & $368.59 \pm 29.14$ & $296.746 \pm 11.27$ & $194.889 \pm 8.20$ \\
T98 & $430.571 \pm 35.97$ & $284.619 \pm 35.88$ & $258.276 \pm 13.85$ \\
U251 & $443.651 \pm 33.51$ & $269.327 \pm 19.37$ & $169.194 \pm 5.77$ \\
LN229 & $417.436 \pm 27.86$ & $332.011 \pm 21.58$ & $276.165 \pm 10.32$ \\
\hline
\end{tabular}

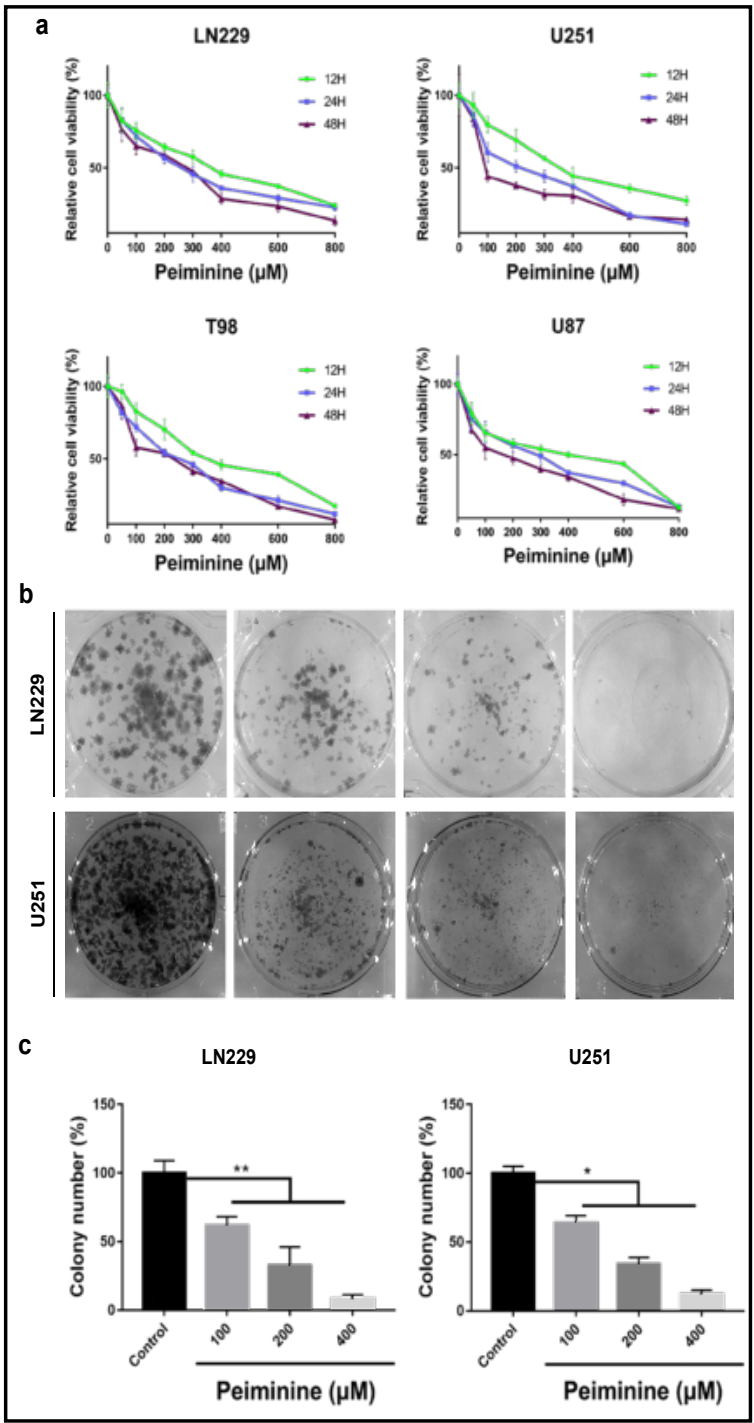

Fig. 1. Peiminine inhibits the proliferation and colony formation of GBM cells (a) GBM cell lines LN229, U251, U87 and T98 were treated with peiminine at concentrations of 0 to $800 \mu \mathrm{M}$ in triplicate for 12,24 and $48 \mathrm{~h}$. Cell viability was measured by MTT assay. (b) U251 and LN229 cells were treated with peiminine at different concentrations $(0,100,200$ or $400 \mu \mathrm{M})$ for 7 days. (c) Bar plot of the average colony number of LN229 and U251 cells. Data are expressed as the mean \pm SD. Compared with the control group: ${ }^{* *}, \mathrm{p}<0.01,{ }^{*}, \mathrm{p}<0.05$. 


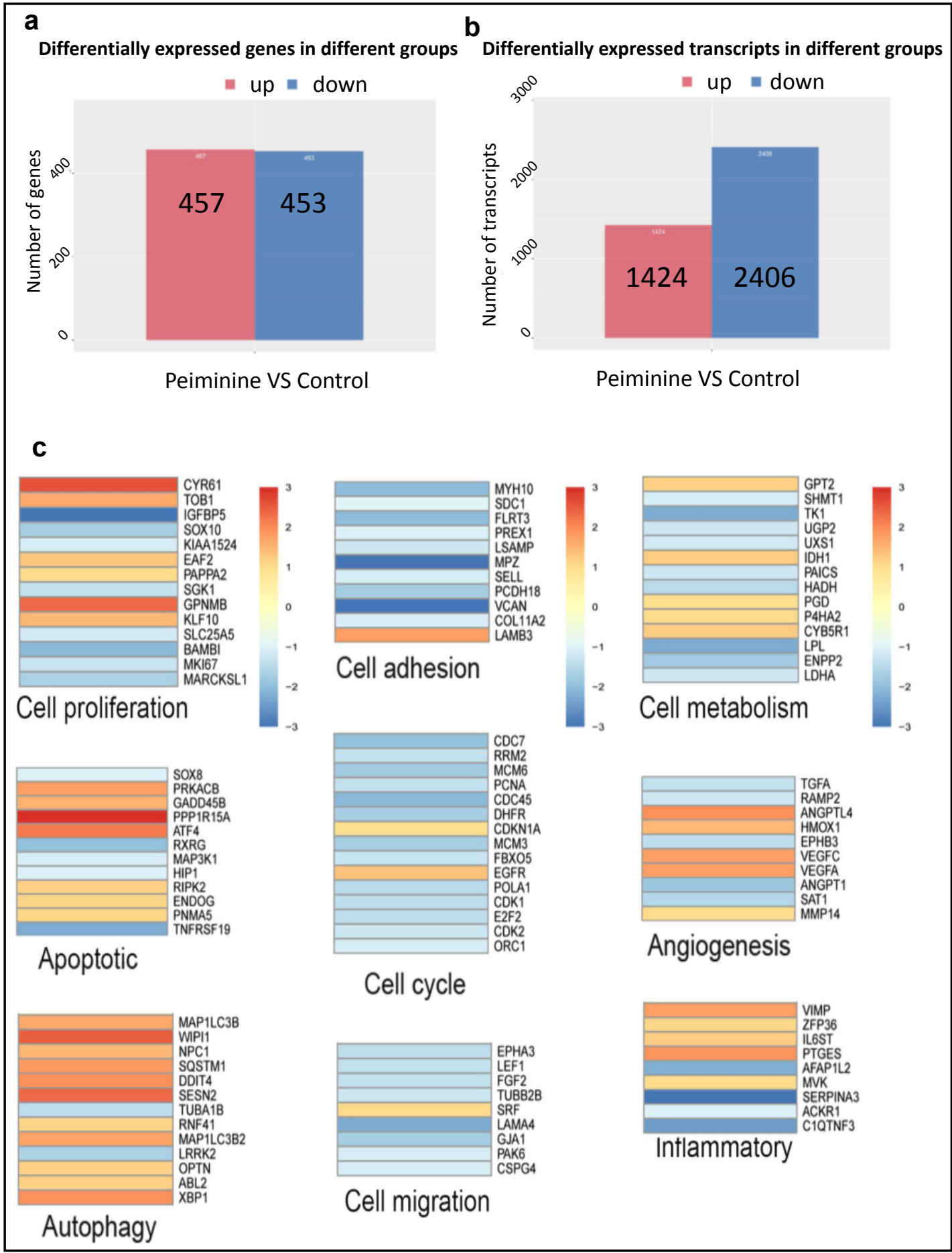

Fig. 2. Altered expression of cancer-associated genes by peiminine in GBM cells (a) The differential expression of genes and transcripts in LN229 cells treated with peiminine (200 $\mathrm{M}, 24-\mathrm{h}$ culture). (b) A subset of the genes altered by peiminine treatment, including genes involved in different aspects of cancer cell development, such as proliferation, cell metabolism, cell cycle, autophagy, apoptosis, cell migration, angiogenesis, cell adhesion and inflammatory response. 
The results of the RNA-seq experiment were in accordance with those of our previous experiments (Fig. 1) examining peiminine-induced inhibition of GBM cell proliferation. Although these differentially expressed genes and transcripts are involved in nearly every facet of GBM cells, the changes in the cell cycle markers and the nutrientsensing kinase signalling were particularly evident. Furthermore, the transformations of autophagy-related molecules, such as SQSTM1/p62 and LRRK2, were different than what we had expected.

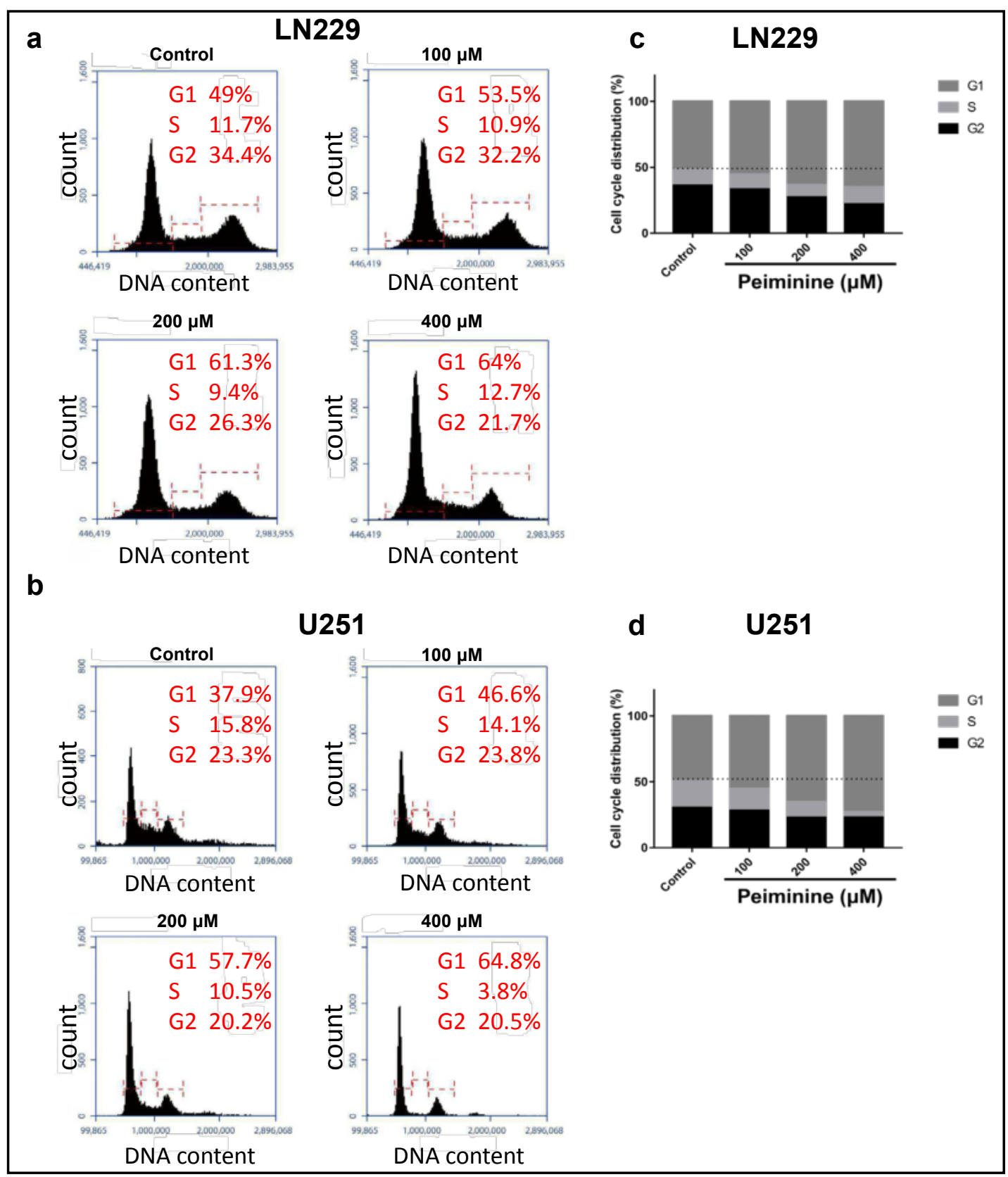

Fig. 3. Peiminine induces cell cycle arrest in GBM cells (a)(b) LN229 and U251 cells were treated with peiminine at different concentrations $(0,100,200$ or $400 \mu \mathrm{M})$ for $24 \mathrm{~h}$, and cell cycle distribution was evaluated after propidium iodide (PI) staining. (c)(d) Bar plot of the cell cycle distribution of LN229 and U251 cells. 
Peiminine induces cell cycle arrest in GBM cells

As shown by the flow cytometry analysis, peiminine caused cell cycle arrest in LN229 and U251 cells at the G1/G0 phase (Fig. 3a, b, c and d). We further performed a separate analysis comparing the differences in G1/G0 phase of the cell cycle. The increase in cells in the G1/G0 phase was clearly statistically significant as shown in Fig. S1 (For all supplemental material see www.karger. com/10.1159/000495646/). Consulting the previous RNAseq data on the cell cycle, we determined that cyclindependent kinase (CDK) inhibitor 1A (p21) and CDK2 had corresponding changes. In addition, to validate the above results, we used Western blotting to assess

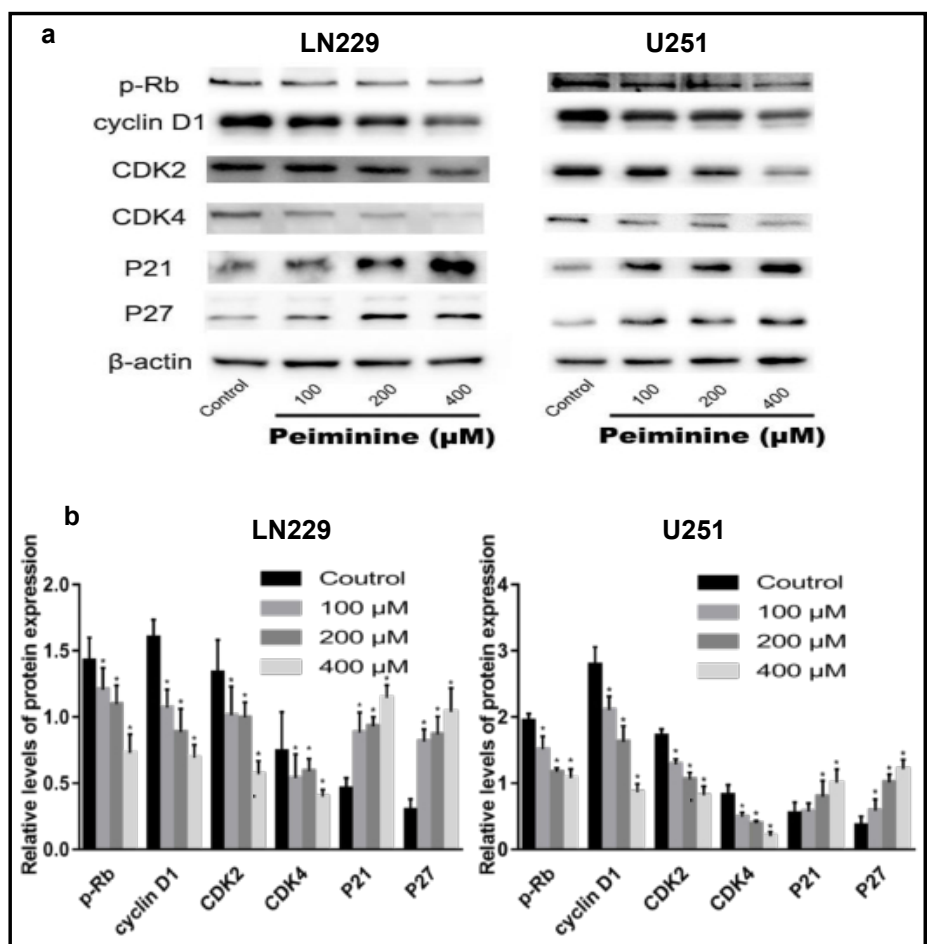

Fig. 4. Peiminine induces cell cycle arrest in GBM cells (a)(b) Expression of cell cycle-related proteins in LN229 and U251 cells as determined by Western blotting. ${ }^{*}, \mathrm{p}<0.05$, compared with the control group. the expression of p21, p27, CDK2, CDK4, cyclin D1 and phosphorylated (p-) retinoblastoma protein $(\mathrm{Rb})$. Peiminine downregulated the expression of cyclin D1, CDK2, CDK4 and p-Rb and upregulated the expression of p21 and p27 (Fig. 4a, b).

Therefore, our data demonstrated that cell cycle arrest was induced by peiminine through the downregulation of CDKs and upregulation of CDK inhibitors in U251 and LN229 cells.

Peiminine induces autophagosome accumulation and blocks autophagic flux in GBM cells

To detect the role of autophagy in peiminine-treated GBM cells, expression of a specific marker of autophagosome accumulation, LC3, was analysed by Western blotting in a dose- and time-dependent manner. Both the LN229 and U251 cell lines showed significant increases in the abundance of LC3-II with peiminine treatment (Fig. 5a, b). Furthermore, transmission electron microscopy (TEM) corroborated that there was an increase in autophagosomes in the cytoplasm following treatment with peiminine compared to the presence of autophagosomes in control cells (Fig. 5c).

The accumulation of autophagosomes may be associated with the reduction in autophagosome degradation. Therefore, we also analysed the degree of expression of p62, an autophagy marker associated with the degradation of autophagosomes. There was a significant time- and dose-dependent increase in p62 expression with peiminine treatment (Fig. 5d, e). Our results thus indicate that peiminine not only caused an accumulation of autophagosome but also supressed the degradation of autophagosomes. These phenomena usually stem from the obstruction of autophagic flux. 


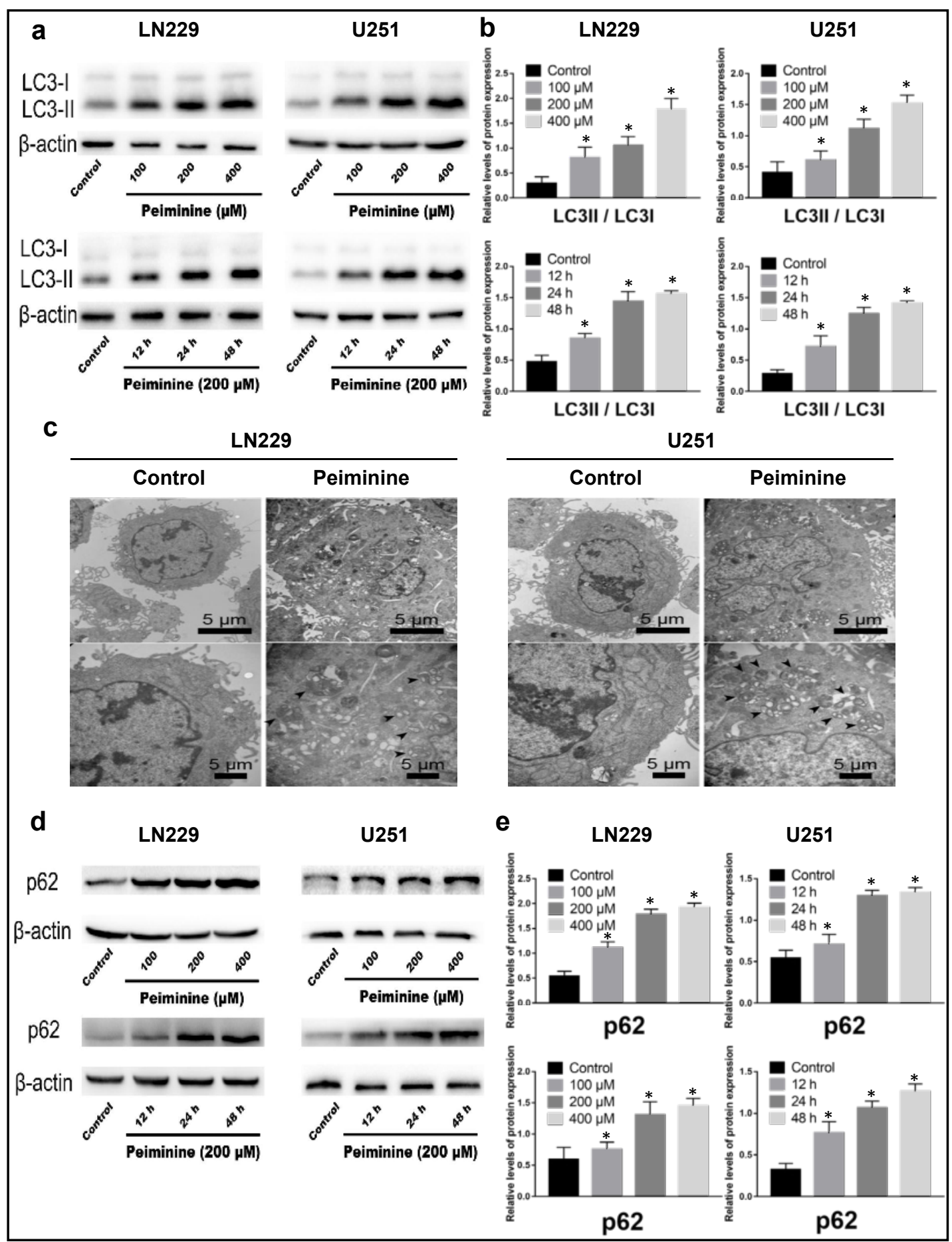

Fig. 5. Peiminine induces autophagosome accumulation and blocks autophagic flux in GBM cells (a)(b) Western blotting analysis of the effects of peiminine on LC3 in a dose- and time-dependent manner. *, p $<0.05$, the LC3II/LC3I value was compared and standardized compared with the control group. (c) LN229 and U251 cells were treated with peiminine $(200 \mu \mathrm{mol} / \mathrm{L})$ for $6 \mathrm{~h}$. Ultrathin sections were cut and examined by transmission electron microscopy. Arrows indicate autophagosomes. (d)(e) Western blotting analysis of p62 in LN229 and U251 cells treated with peiminine in a dose- and time-dependent manner. *, p<0.05, compared with the control group. 
Molecular alteration of AKT and AMPK signalling is responsible in LN229 and U251 cells

The RNA-seq experiment revealed a number of changes linked to nutrient-sensing kinase signalling, including the AKT and AMPK pathways (Fig. 6a). Cyclin D1 is regulated by the AKT-GSK3 $\beta$ pathway [22], and cyclin D1 protein expression is downregulated by peiminine treatment in GBM cells, as shown in our previous experiment. The autophagyrelated factor p62/SQSTM1 is mainly regulated by ULK1, which is affected by both AKT and AMPK in response to energetic stress [18]. These are indications that the AKT and AMPK signalling pathways might play important roles in GBM inhibition by peiminine. After $24 \mathrm{~h}$ of peiminine treatment in LN229 and U251 cells, AKT phosphorylation was obviously reduced compared to that in the control group, and simultaneously, PI3K and GSK3 $\beta$, which are the essential upstream and downstream effectors, were dephosphorylated. In addition, the

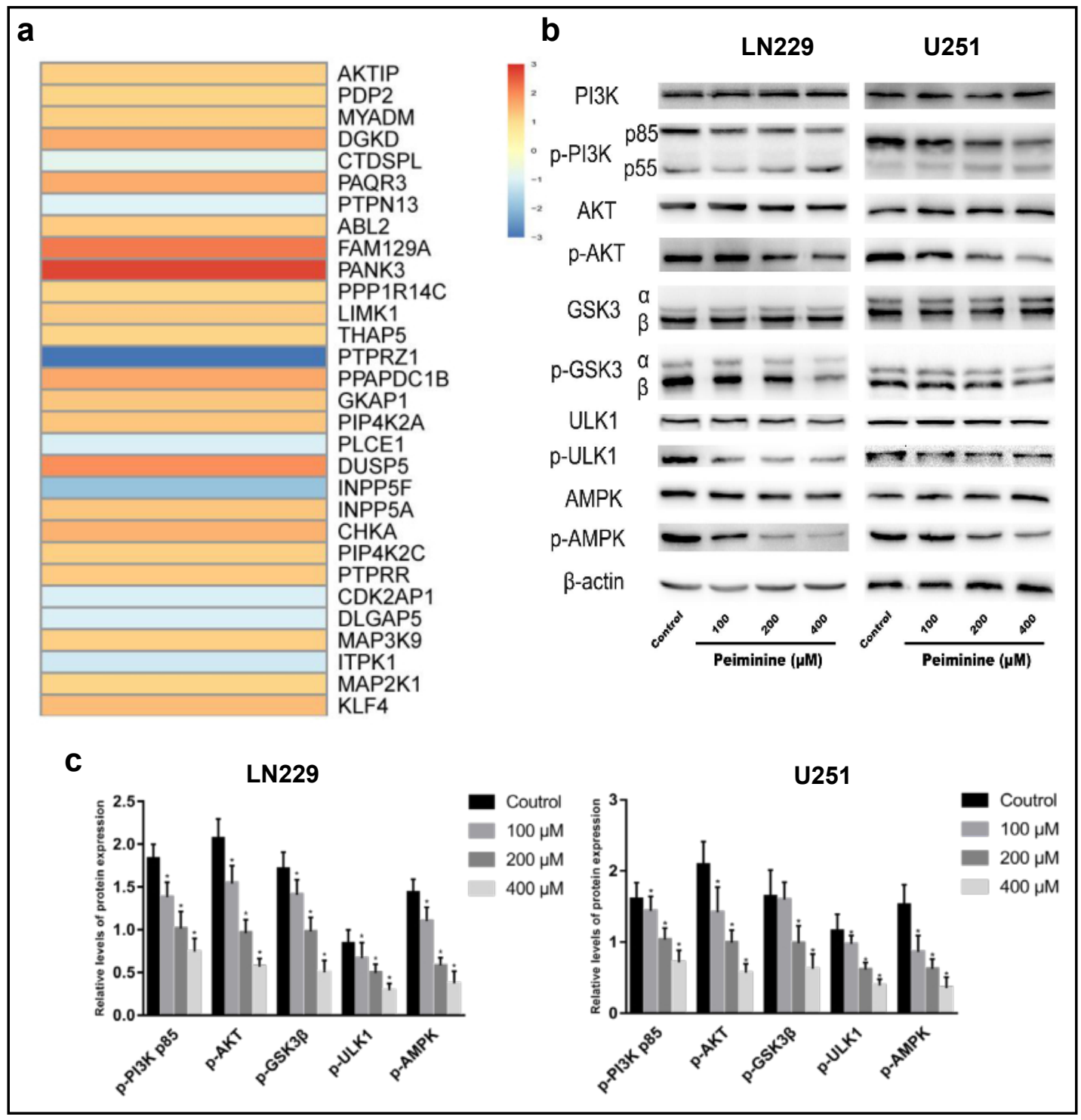

Fig. 6. Molecular alteration of AKT and AMPK signalling plays a role in LN229 and U251 cells (a) A subset of the altered genes identified by RNA-seq that are linked to nutrient-sensing kinase signalling, including the AKT and AMPK pathways. (b)(c) LN229 and U251 cells were treated with different concentrations of peiminine for $24 \mathrm{~h}$. Then, the cells were collected and lysed. Western blotting was performed by probing with anti-PI3K, anti-phospho-PI3K, anti-AKT, anti-phospho-AKT, anti-GSK3 $\alpha / \beta$, anti-phospho-GSK3 $\alpha / \beta$, anti-ULK1 and anti-phospho-ULK1. *, p<0.05, compared with the control group. 
treatment downregulated P-AMPK and P-ULK1 levels in a peiminine concentration-related manner (Fig. 6b, c). These results suggested that alterations in AKT-GSK3 $\beta$ and AMPK-ULK1 signalling are responsible for the changes in cell cycle and autophagic status in LN229 and U251 cells.

Peiminine induces cell cycle arrest and blocking of autophagic flux in GBM cells by inhibiting the AKT-GSK3 $\beta$ and AMPK-ULK1 pathways

Next, we verified whether the AKT-GSK3 $\beta$ and AMPK pathways were involved in the effects of peiminine on cell cycle arrest and autophagic flux blocking. LN229 and U251 cells were transfected with a lentivirus to overexpress AKT (Lv-AKT). The Lv-AKT cells showed decreased LC3-II expression and increased p62 and cyclin D1 expression; furthermore, the levels of LC3-II and p62 were enhanced, and cyclin D1 expression was reduced by peiminine treatment (Fig. 7a, b). Moreover, in the cells that were preconditioned with LY294002, a morpholine-containing compound that is a potent inhibitor of the AKT pathway [23], there were also increases in the LC3-II expression level but decreases in the p62 expression level. The downregulation of cyclin D1 was also more obvious in this group (Fig. 8a, b).

Metformin hydrochloride (Met), an AMPK activator, was also used to assess the role of AMPK in peiminine-mediated autophagic flux blocking. As shown in Fig. 8c and d, Western blot analysis revealed that changes in the expression of p62 induced by peiminine treatment were reversed by the addition of Met.

These observations indicated that the AKT-GSK3 $\beta$ and AMPK-ULK1 signalling pathways are involved in peiminine-induced cell cycle arrest and autophagic flux blocking in GBM cells.

Fig. 7. Peiminine induces cell cycle arrest and blocking of autophagic flux in GBM cells by inhibiting the AKTGSK3 $\beta$ and AMPK pathways (a)(b) LN229 and U251 cells transfected with a lentivirus overexpressing AKT (Lv$\mathrm{AKT}$ ) and virus without AKT (Lv-control) were treated with peiminine for $24 \mathrm{~h}$. Western blot analysis was performed to assess levels of AKT, phosphoAKT, GSK3 $\alpha / \beta$, phosphoGSK3 $\alpha / \beta$, ULK1, phosphoULK1, p62, cyclin D1 and LC3. **, $\mathrm{p}<0.01, *, \mathrm{p}<0.05$, compared with the control group.

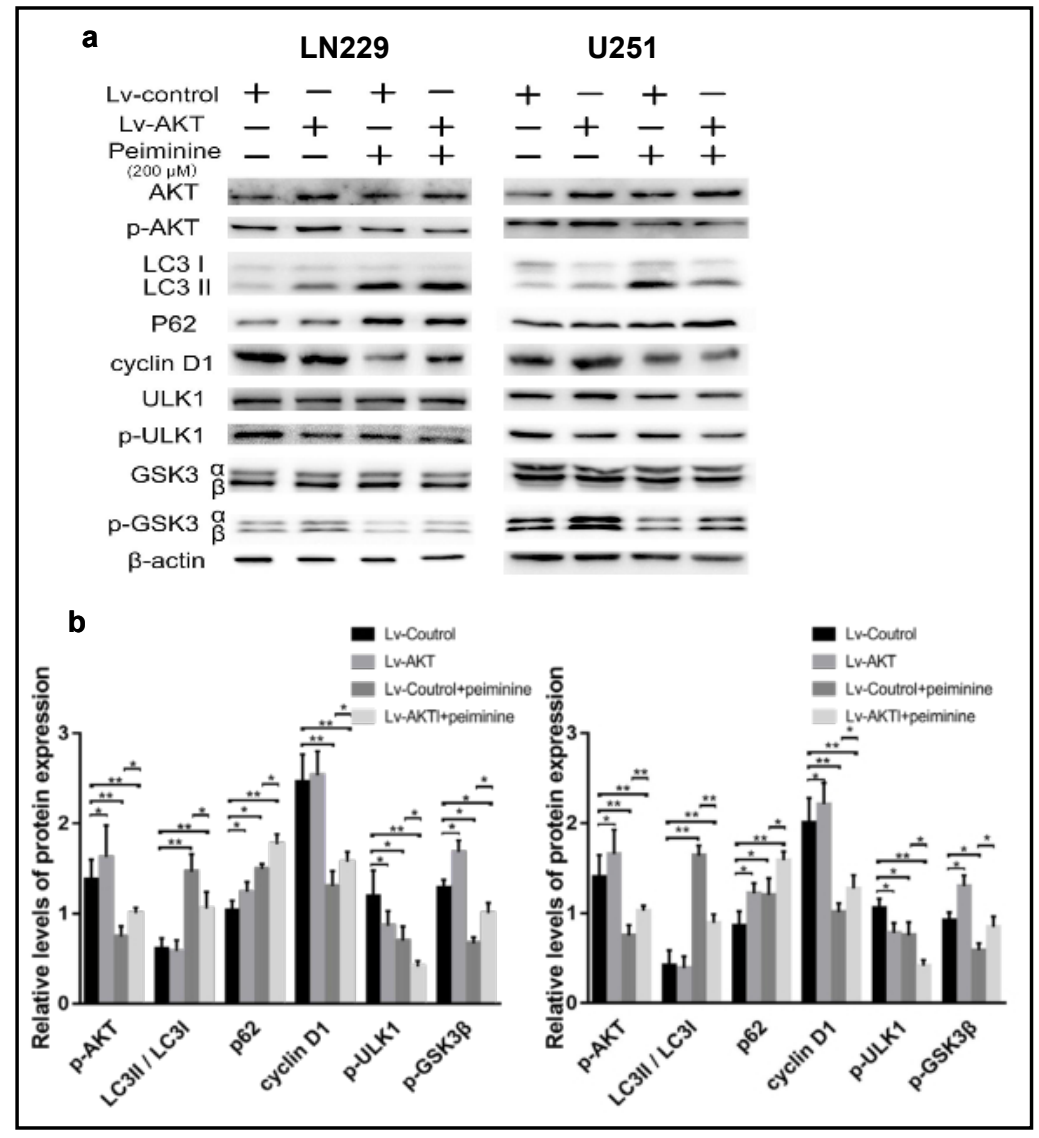


Fig. 8. Peiminine induces cell cycle arrest and blocking of autophagic flux in GBM cells by inhibiting the AKT-GSK3 $\beta$ and AMPK pathways (a) (b) LN229 and U251 cells were incubated with 10 $\mu \mathrm{M}$ LY294002, either alone or in combination with $200 \mu \mathrm{M}$ peiminine, for 24 $\mathrm{h}$, and then Western blot analysis was performed to assess levels of AKT, phospho-AKT, GSK3 $\alpha / \beta$, phospho-GSK3 $\alpha / \beta$, ULK1, phospho-ULK1, p62, cyclin D1 and LC3. **, p<0.01, *, $\mathrm{p}<0.05$, compared with the control group. (c)(d) LN229 and U251 cells were pretreated with 2 $\mu \mathrm{M}$ Met, either alone or in combination with 200 $\mu \mathrm{M}$ peiminine, for $24 \mathrm{~h}$, and then Western blot analysis was performed to assess levels of AMPK, phospho-AMPK, p62 and LC3. ${ }^{* *}, \mathrm{p}<0.01, *, \mathrm{p}<0.05$, compared with the control group.

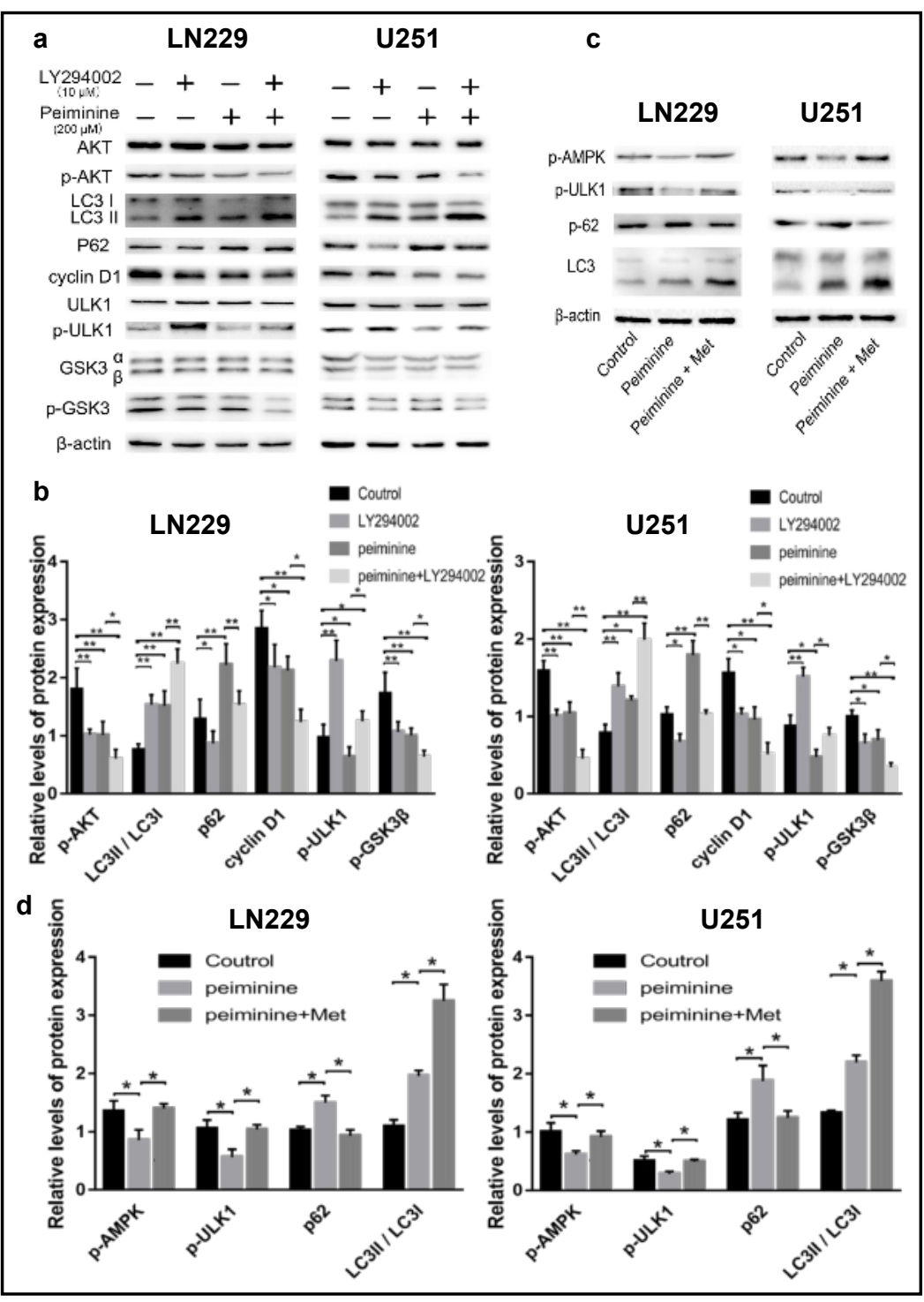

Peiminine inhibits GBM growth in vivo

Our previous experiments have shown that peiminine has an antitumour effect on GBM cells in vitro. To evaluate whether peiminine also has an anti-GBM effect in vivo, we established a xenograft tumour model of human GBM by subcutaneous injection of U251 cells in nude mice. Peiminine treatment inhibited the tumour growth (Fig. 9a and b). Furthermore, the immunohistochemical (IHC) and Western blotting data suggested that there are the same mechanisms of anti-tumourigenesis in vitro and in vivo (Fig. 9c and d).

In summary, our results confirmed that peiminine could also inhibit GBM cell growth through cell cycle arrest and autophagic flux blocking in vivo. 


\section{Cellular Physiology

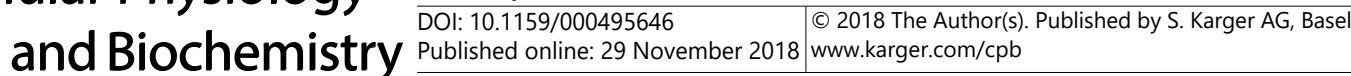

Fig. 9. Peiminine inhibits GBM growth in vivo (a) U251 xenograft tumours in different groups at the end of the experiments. (b) Tumour weight in different groups. (c) Immunohistochemical staining for LC3, P62 and cyclin D. Images were obtained at a magnification of $400 \mathrm{x}$ (length of $50 \mu \mathrm{m}$ ) (d) Phospho-AKT, phosphoAMPK, phospho-ULK1, cyclin D1, P62 and LC3 levels were examined by Western blot. *, $\mathrm{p}<0.05$, compared with the control group.

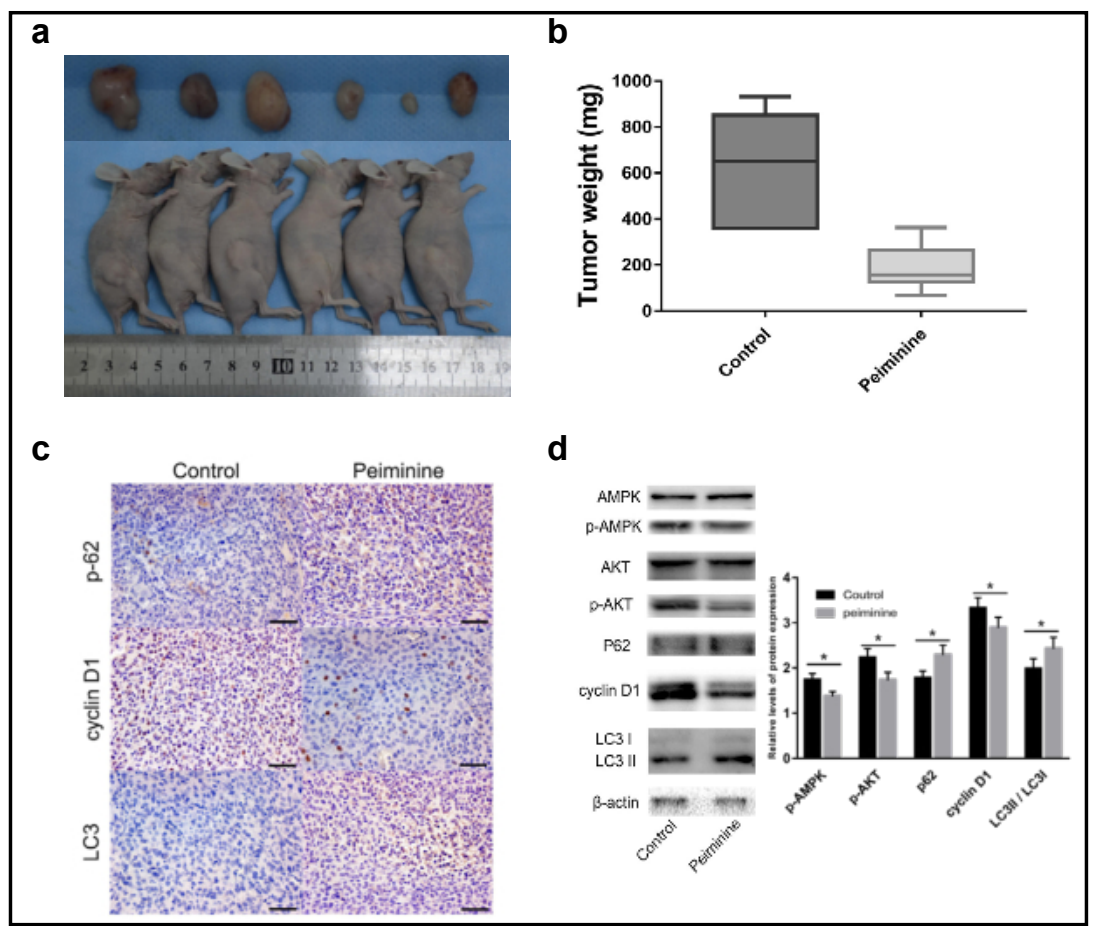

\section{Discussion}

GBM, which is defined as a grade IV glioma by the WHO, is invariably accompanied by rapid clinical deterioration and death in patients $[1,2]$. Even when combination therapies of maximum surgical excision, radiotherapy and adjuvant temozolomide treatment are applied, the median survival of GBM patients is still less than 15 months [3, 24]. Therefore, more complementary treatment strategies are necessary. For the last several years, traditional Chinese medicine has demonstrated its efficacy as an alternative medicine to treat many cancers, including GBM [5, 6, 25]. Peiminine, an alkaloid derived from Fritillaria thunbergii, has been reported to have potential antitumour effects [1,26]. Additionally, it has been previously demonstrated that peiminine plays a role in protecting dopaminergic neurons in a rat model by inhibiting neuroinflammation [27]. These findings confirmed that peiminine could cross the blood-brain barrier. Thus, the current study aimed to verify whether peiminine has GBM-combating properties and to determine its anticarcinogenic mechanism so that it can be developed into a new treatment option for this devastating cancer.

First, the results of the present study indicate that peiminine inhibited GBM cell viability in vitro and in vivo (Fig. 1a-d, Fig. 9a and b). Subsequently, our RNA-seq data of LN229 cells that were treated with peiminine identified many cancer-associated changes in gene and transcript expression levels (Fig. 2c). The differential expression of several cell cycle genes was particularly obvious, and it was also interesting that the autophagy-associated changes were not exactly the same as those reported in other studies on peiminine in colorectal carcinoma [13].

Then, we demonstrated that peiminine induced cell cycle arrest in LN229 and U251 cells at the G1/G0 phase (Fig. 3a-d). It is not surprising that cell cycle arrest, which is usually representative of a cancer cell response to DNA damage, is a component of most therapeutic modalities for cancer [28]. Furthermore, using RNA-seq and Western blot analyses, we observed clear changes in cell cycle-related genes, among which the cyclin D1 gene commonly observed in human cancer cells and strongly associated with cell proliferation in numerous malignancies is one of the most commonly observed markers of cell cycle control regulation that governs the transition through the G1 phase $[29,30]$. Cyclin D1 can bind and 


\section{Cellular Physiology Cell Physiol Biochem 2018;51:1566-1583 \\ and BiOChemistry \begin{tabular}{l|l} 
DOI: $10.1159 / 000495646$ & $\begin{array}{l}\text { C } 2018 \text { The Author(s). Published by S. Karger AG, Basel } \\
\text { www.karger.com/cpb }\end{array}$
\end{tabular} \\ Zhao et al.: Antitumour Effect of Peiminine on Glioblastoma}

activate CDK4, which then phosphorylates Rb. These are very important parts of the G1/G0 transition in the cell cycle [31, 32]. In addition, p21 and p27, as inhibitors of cyclin D1/CDK complex activation, have been reported to inhibit DNA replication and block the G1/G0 cell cycle transition in cancer cells $[33,34]$. Our results suggest that peiminine induces G1/G0 phase arrest by increasing the expression of p21 and p27 and decreasing the expression of cyclin D1, CDK2 and CDK4.

Autophagy is a double-edged sword in tumour progression and treatment: it can either facilitate cancer survival under metabolic stress or induce autophagic tumour cell death [35, 36]. Mounting evidence accumulated in recent years suggests that inhibition of autophagy can reduce the treatment resistance of chemotherapy and radiotherapy in various types of cancers $[37,38]$, which is also the case in GBM treatment $[39,40]$. To clarify the role of autophagy in peiminine therapy for GBM, LN229 and U251 cells were observed under TEM after peiminine treatment (Fig. 5c), and we also used Western blotting to detect LC3 and p62 expression of which LC3 (Atg8) is a key protein in autophagy, which functions primarily in the cytoplasm where the autophagosomes and autolysosomes exist [41, 42], and another widely used autophagy marker, p62, serves as a marker of autophagosome degradation [43] (Fig. 5a, b, d and e). Unlike previous research on peiminine, this study tested autophagy by combining the above experiments, and we observed an upregulation of p62 expression. Our results indicated a role of autophagy in peiminine therapy for GBM, suggesting that peiminine treatment leads to autophagosome accumulation and blocks autophagic flux in GBM cells. Thus, peiminine might also be a promising potential adjuvant therapy for GBM that can be used to overcome chemotherapy and radiation resistance.

To further understand the antineoplastic mechanisms of peiminine in GBM cells, we screened the results of the RNA-seq analysis and searched for a correlation between the previous experimental results and the status of various oncogenic signalling cascades. In addition to the changes in cell cycle and autophagy, the RNA-seq experiment revealed a number of changes linked to nutrient-sensing kinase signalling, including the AKT and AMPK pathways. AKT not only stabilizes cyclin D1 by phosphorylation and inactivation of GSK3 $\beta$ but also directly inhibits p21 and p27 [33, 34]. Moreover, as a downstream effector, GSK3 $\beta$ can also regulate cyclin D1 $[15,22,44]$, while GSK3 $\beta$ inhibition increases the level of p21 and downregulates CDK and p-RB, which can block cell cycle progression [32, 33]. In this study, we demonstrated that peiminine significantly inhibits phosphorylation of AKT and GSK3 $\beta$ in GBM cell lines (Fig. 6b and c). Peiminine-mediated cell cycle arrest of GBM cells was confirmed by transfecting LV-AKT cells, LN229 and U251 cells with a lentivirus overexpressing AKT. Overexpression of AKT led to a reversal of cell cycle arrest, as evidenced by increased levels of cyclin D1 and p-GSK3 $\beta$. (Fig. 7a and b) In addition, LY294002, a morpholine-containing compound that is a potent inhibitor of the AKT pathway [23], produced similar effects as peiminine, further confirming that peiminine inhibits AKT/GSK3 $\beta$ to induce cell cycle arrest in GBM cells (Fig. 8a and b). Our data indicated that the induction of cell cycle arrest in LN229 and U251 cells by peiminine was due to suppress the AKT-GSK3 $\beta$ signalling pathway.

Increasing evidence demonstrates that the autophagy receptor protein p62/SQSTM1 is mainly mediated through ULK1 in response to energetic stress [45]. In addition, it is known that AKT regulates ULK1 during the process of autophagy, which is also regulated by AMPK [18, 20]. Akt signalling enhances autophagy by promoting ULK1 phosphorylation [20]. However, AMPK has also been shown to phosphorylate and activate ULK1 during autophagy $[18,19]$. Although evidence indicates that AKT and AMPK are associated with autophagy, autophagy is a dynamic process, and there may be many other potential regulatory mechanisms. For instance, some researchers theorize that ULK1 also participates in feedback mechanisms, which may allow for additional control $[46,47]$. However, the interaction between AKT/ AMPK/ULK1 and its relationship with autophagy are still not clear, and the network of relationships between them requires further study. In this study, we demonstrated that peiminine significantly inhibits the phosphorylation of AKT, AMPK and ULK1 in GBM cell lines (Fig. 6b and c). However, in autophagy, the inhibition of these two pathways will generally produce very different results. Inhibition of AMPK signals can lead to reversal 


\section{Cellular Physiology

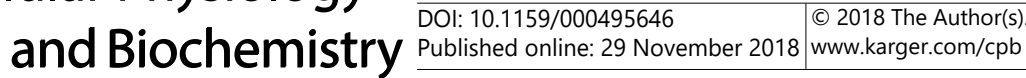

of autophagy by suppressing the Akt-mTOR signalling pathway [21]. Similarly, our results imply that when there is competition for ULK1 as the substrate under peiminine conditions in GBM cells, the effects on the AMPK-ULK1 pathway are greater. Furthermore, in AKT-overexpressing cells and AKT suppressor cells, peiminine antagonizes ULK1mediated autophagy by inhibiting AMPK signalling (Fig. 7a, b and Fig. 8a, b). Peiminine-mediated AMPKdependent autophagic flux blocking was also confirmed by Met, an AMPK activator. In the activated AMPK group, autophagy was reversed, as evidenced by the decreased expression of p62 (Fig. 8c and d).

In summary, we demonstrated

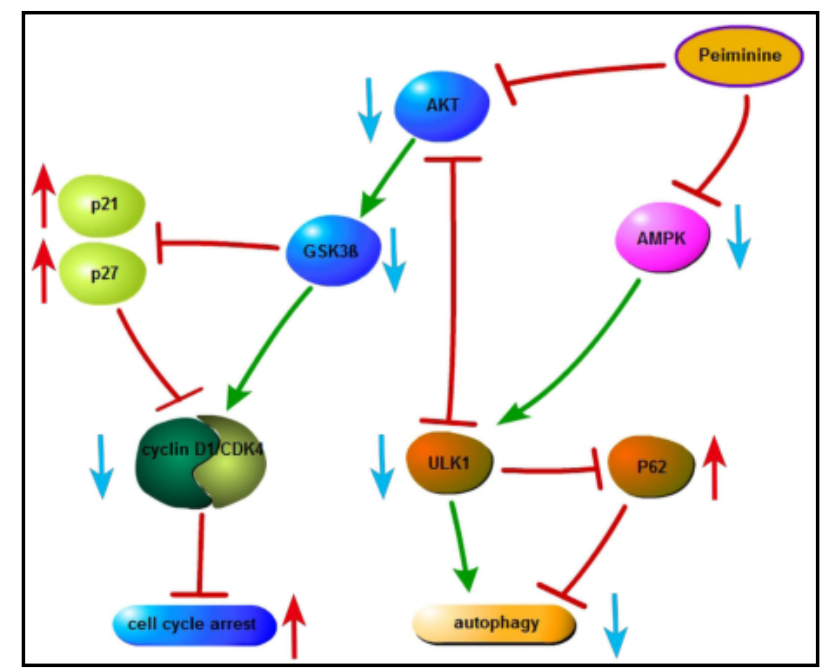

Fig. 10. Proposed mechanistic signalling pathways for AKTGSK3 $\beta$ cell cycle arrest and AMPK-ULK1 autophagy inhibition in GBM cells in response to treatment with peiminine. for the first time the anticancer properties of peiminine in GBM cells both in vitro and in vivo. In addition, as a different mechanism from that of peiminine in colon cancer, we clearly demonstrated that peiminine inhibits GBM cell growth through induction of the G1/G0 phase cell cycle arrest and autophagic flux blocking, which appears to be regulated via the AKT-GSK3 $\beta$ and AMPK signalling pathways. Also, peiminine may also have excellent adjuvant chemotherapy effect in GBM cells. We have did some preliminary experiments shown that peiminine can enhance the anti-GBM effect of temozolomide (as shown in Fig. S2). Therefore, peiminine could provide a new anti-GBM treatment and deserves further study in additional in vivo experiments and clinical trials.

\section{Conclusion}

Our work shows, as illustrated in Fig. 10, that peiminine inhibits glioblastoma in vitro and in vivo through cell cycle arrest by suppressing Akt-GSK3 $\beta$ signalling and autophagic flux blocking by depressing AMPK/ULK1 signalling.

\section{Abbreviations}

GBM (Glioblastoma multiforme); WHO (World Health Organization); TEM (transmission electron microscopy); AKT (protein kinase B); GSK3 $\beta$ (glycogen synthase kinase $3 \beta$ ); AMPK (AMP-activated protein kinase); DMEM (Dulbecco's Modified Eagle's Medium); FBS (foetal bovine serum); DMSO (dimethyl sulfoxide); BCA (radiobicinchoninic acid); RIPA (immunoprecipitation assay); RNA-seq (transcriptome shotgun sequencing); $\mathrm{IC}_{50}$ (inhibitory concentration); CDK (cyclin-dependent kinase); p- (phosphorylated); Rb (retinoblastoma protein); Lv-AKT (transfected with a lentivirus to overexpress AKT); Met (metformin hydrochloride); IHC (immunohistochemical). 


\section{Cellular Physiology Cell Physiol Biochem 2018;51:1566-1583

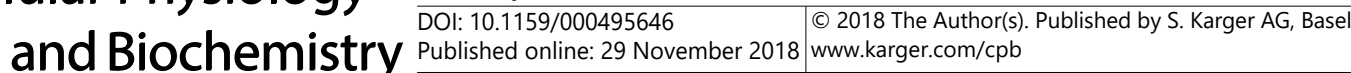 \\ Zhao et al.: Antitumour Effect of Peiminine on Glioblastoma}

\section{Acknowledgements}

This study was primarily supported by the Scientific Fund of Heilongjiang Province for Youth (QC2016124), the First Affiliated Hospital of Harbin Medical University Foundation (2016B006) and the Special Fund for Translational Research of the Sino-Russia Medical Research Centre of Harbin Medical University (CR201410).

\section{Disclosure Statement}

The authors declared no potential conflicts of interest with respect to the research, authorship, and/or publication of this article.

\section{References}

1 Louis DN, Perry A, Reifenberger G, von Deimling A, Figarella-Branger D, Cavenee WK, Ohgaki H, Wiestler OD, Kleihues P, Ellison DW: The 2016 World Health Organization Classification of Tumors of the Central Nervous System: a summary. Acta Neuropathol 2016;131:803-820.

-2 Wen PY, Kesari S: Malignant gliomas in adults. N Engl J Med 2008;359:492-507.

-3 Stupp R, Hegi ME, Mason WP, van den Bent MJ, Taphoorn MJ, Janzer RC, Ludwin SK, Allgeier A, Fisher B, Belanger K, Hau P, Brandes AA, Gijtenbeek J, Marosi C, Vecht CJ, Mokhtari K, Wesseling P, European Organisation for R, Treatment of Cancer Brain T, Radiation Oncology G, National Cancer Institute of Canada Clinical Trials $\mathrm{G}$ et al.: Effects of radiotherapy with concomitant and adjuvant temozolomide versus radiotherapy alone on survival in glioblastoma in a randomised phase III study: 5-year analysis of the EORTC-NCIC trial. Lancet Oncol 2009;10:459-466.

-4 Wen PY, Reardon DA: Neuro-oncology in 2015: Progress in glioma diagnosis, classification and treatment. Nat Rev Neurol 2016;12:69-70.

5 Zhong C, Wall NR, Zu Y, Sui G: Therapeutic Application of Natural Medicine Monomers in Cancer Treatment. Curr Med Chem 2017;24:3681-3697.

6 Wang CY, Bai XY, Wang CH: Traditional Chinese medicine: a treasured natural resource of anticancer drug research and development. Am J Chin Med 2014;42:543-559.

7 Ruan X, Yang L, Cui WX, Zhang MX, Li ZH, Liu B, Wang Q: Optimization of Supercritical Fluid Extraction of Total Alkaloids, Peimisine, Peimine and Peiminine from the Bulb of Fritillaria thunbergii Miq, and Evaluation of Antioxidant Activities of the Extracts. Materials (Basel) 2016;9.

-8 Hao DC, Gu XJ, Xiao PG, Peng Y: Phytochemical and biological research of Fritillaria medicine resources. Chin J Nat Med 2013;11:330-344.

-9 Wu K, Mo C, Xiao H, Jiang Y, Ye B, Wang S: Imperialine and Verticinone from Bulbs of Fritillaria wabuensis Inhibit Pro-inflammatory Mediators in LPS-stimulated RAW 264.7 Macrophages. Planta Med 2015;81:821829.

10 Chan SW, Li SL, Lin G, Li P: Pharmacokinetic study and determination of imperialine, the major bioactive component in antitussive Fritillaria cirrhosa, in rat by high-performance liquid chromatography coupled with evaporative light-scattering detector. Anal Biochem 2000;285:172-175.

11 Xu F, Xu S, Wang L, Chen C, Zhou X, Lu Y, Zhang H: Antinociceptive efficacy of verticinone in murine models of inflammatory pain and paclitaxel induced neuropathic pain. Biol Pharm Bull 2011;34:1377-1382.

12 Guo H, Ji F, Liu B, Chen X, He J, Gong J: Peiminine ameliorates bleomycin-induced acute lung injury in rats. Mol Med Rep 2013;7:1103-1110.

13 Lyu Q Tou F, Su H, Wu X, Chen X, Zheng Z: The natural product peiminine represses colorectal carcinoma tumor growth by inducing autophagic cell death. Biochem Biophys Res Commun 2015;462:38-45.

14 Laurent PA, Severin S, Gratacap MP, Payrastre B: Class I PI 3-kinases signaling in platelet activation and thrombosis: PDK1/Akt/GSK3 axis and impact of PTEN and SHIP1. Adv Biol Regul 2014;54:162-174.

15 Majewska E, Szeliga M: AKT/GSK3beta Signaling in Glioblastoma. Neurochem Res 2017;42:918-924. 


\section{Cellular Physiology Cell Physiol Biochem 2018;51:1566-1583

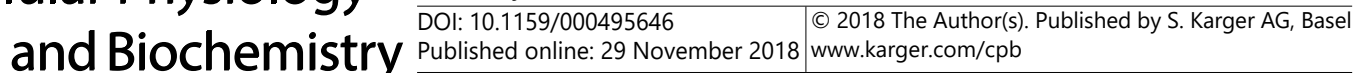

16 Bernardi A, Frozza RL, Hoppe JB, Salbego C, Pohlmann AR, Battastini AM, Guterres SS: The antiproliferative effect of indomethacin-loaded lipid-core nanocapsules in glioma cells is mediated by cell cycle regulation, differentiation, and the inhibition of survival pathways. Int J Nanomedicine 2013;8:711-728.

17 Atkins RJ, Dimou J, Paradiso L, Morokoff AP, Kaye AH, Drummond KJ, Hovens CM: Regulation of glycogen synthase kinase-3 beta (GSK-3beta) by the Akt pathway in gliomas. J Clin Neurosci 2012;19:1558-1563.

18 Hardie DG, Ross FA, Hawley SA: AMPK: a nutrient and energy sensor that maintains energy homeostasis. Nat Rev Mol Cell Biol 2012;13:251-262.

-19 Egan DF, Shackelford DB, Mihaylova MM, Gelino S, Kohnz RA, Mair W, Vasquez DS, Joshi A, Gwinn DM, Taylor R, Asara JM, Fitzpatrick J, Dillin A, Viollet B, Kundu M, Hansen M, Shaw RJ: Phosphorylation of ULK1 (hATG1) by AMP-activated protein kinase connects energy sensing to mitophagy. Science 2011;331:456461.

20 Bach M, Larance M, James DE, Ramm G: The serine/threonine kinase ULK1 is a target of multiple phosphorylation events. Biochem J 2011;440:283-291.

-21 Zhang P, Lai ZL, Chen HF, Zhang M, Wang A, Jia T, Sun WQ, Zhu XM, Chen XF, Zhao Z, Zhang J: Curcumin synergizes with 5-fluorouracil by impairing AMPK/ULK1-dependent autophagy, AKT activity and enhancing apoptosis in colon cancer cells with tumor growth inhibition in xenograft mice. J Exp Clin Cancer Res 2017;36:190.

22 Takahashi-Yanaga F, Sasaguri T: GSK-3beta regulates cyclin D1 expression: a new target for chemotherapy. Cell Signal 2008;20:581-589.

23 Maira SM, Stauffer F, Schnell C, Garcia-Echeverria C: PI3K inhibitors for cancer treatment: where do we stand? Biochem Soc Trans 2009;37:265-272.

24 Aldape K, Zadeh G, Mansouri S, Reifenberger G, von Deimling A: Glioblastoma: pathology, molecular mechanisms and markers. Acta Neuropathol 2015;129:829-848.

-25 Hong M, Wang N, Tan HY, Tsao SW, Feng Y: MicroRNAs and Chinese Medicinal Herbs: New Possibilities in Cancer Therapy. Cancers (Basel) 2015;7:1643-1657.

-26 Wang DD, Feng Y, Li Z, Zhang L, Wang S, Zhang CY, Wang XX, Liu ZY: In vitro and in vivo antitumor activity of Bulbus Fritillariae Cirrhosae and preliminary investigation of its mechanism. Nutr Cancer 2014;66:441452.

27 Chen G, Liu J, Jiang L, Ran X, He D, Li Y, Huang B, Wang W, Liu D, Fu S: Peiminine Protects Dopaminergic Neurons from Inflammation-Induced Cell Death by Inhibiting the ERK1/2 and NF-kappaB Signalling Pathways. Int J Mol Sci 2018;19.

28 Kastan MB, Bartek J: Cell-cycle checkpoints and cancer. Nature 2004;432:316-323.

29 Diehl JA: Cycling to cancer with cyclin D1. Cancer Biol Ther 2002;1:226-231.

-30 Musgrove EA, Caldon CE, Barraclough J, Stone A, Sutherland RL: Cyclin D as a therapeutic target in cancer. Nat Rev Cancer 2011;11:558-572.

31 Mittnacht S: Control of pRB phosphorylation. Curr Opin Genet Dev 1998;8:21-27.

-32 Lee Y, Dominy JE, Choi YJ, Jurczak M, Tolliday N, Camporez JP, Chim H, Lim JH, Ruan HB, Yang X, Vazquez F, Sicinski P, Shulman GI, Puigserver P: Cyclin D1-Cdk4 controls glucose metabolism independently of cell cycle progression. Nature 2014;510:547-551.

-33 Jain MV, Jangamreddy JR, Grabarek J, Schweizer F, Klonisch T, Cieslar-Pobuda A, Los MJ: Nuclear localized Akt enhances breast cancer stem-like cells through counter-regulation of p21(Waf1/Cip1) and p27(kip1). Cell Cycle 2015;14:2109-2120.

34 Cheng M, Olivier P, Diehl JA, Fero M, Roussel MF, Roberts JM, Sherr CJ: The p21(Cip1) and p27(Kip1) CDK 'inhibitors' are essential activators of cyclin D-dependent kinases in murine fibroblasts. EMBO J 1999;18:1571-1583.

35 Wang Z, Shi X, Li Y, Fan J, Zeng X, Xian Z, Wang Z, Sun Y, Wang S, Song P, Zhao S, Hu H, Ju D: Blocking autophagy enhanced cytotoxicity induced by recombinant human arginase in triple-negative breast cancer cells. Cell Death Dis 2014;5:e1563.

-36 Yang A, Kimmelman AC: Inhibition of autophagy attenuates pancreatic cancer growth independent of TP53/TRP53 status. Autophagy 2014;10:1683-1684.

37 Gewirtz DA: An autophagic switch in the response of tumor cells to radiation and chemotherapy. Biochem Pharmacol 2014;90:208-211. 


\section{Cellular Physiology Cell Physiol Biochem 2018;51:1566-1583 and Biochemistry DOl: 10.1159/000495646 2018 O 2018 The Author(s). Published by S. Karger AG, Basel

-38 Paglin S, Hollister T, Delohery T, Hackett N, McMahill M, Sphicas E, Domingo D, Yahalom J: A novel response of cancer cells to radiation involves autophagy and formation of acidic vesicles. Cancer Res 2001;61:439444.

39 Kanzawa T, Germano IM, Komata T, Ito H, Kondo Y, Kondo S: Role of autophagy in temozolomide-induced cytotoxicity for malignant glioma cells. Cell Death Differ 2004;11:448-457.

40 Palumbo S, Tini P, Toscano M, Allavena G, Angeletti F, Manai F, Miracco C, Comincini S, Pirtoli L: Combined EGFR and autophagy modulation impairs cell migration and enhances radiosensitivity in human glioblastoma cells. J Cell Physiol 2014;229:1863-1873.

41 Eskelinen EL, Reggiori F, Baba M, Kovacs AL, Seglen PO: Seeing is believing: the impact of electron microscopy on autophagy research. Autophagy 2011;7:935-956.

42 Huang R, Liu W: Identifying an essential role of nuclear LC3 for autophagy. Autophagy 2015;11:852-853.

43 Pankiv S, Clausen TH, Lamark T, Brech A, Bruun JA, Outzen H, Overvatn A, Bjorkoy G, Johansen T: p62/ SQSTM1 binds directly to Atg8/LC3 to facilitate degradation of ubiquitinated protein aggregates by autophagy. J Biol Chem 2007;282:24131-24145.

44 Blume-Jensen P, Hunter T: Oncogenic kinase signalling. Nature 2001;411:355-365.

45 Ro SH, Semple IA, Park H, Park H, Park HW, Kim M, Kim JS, Lee JH: Sestrin2 promotes Unc-51-like kinase 1 mediated phosphorylation of p62/sequestosome-1. FEBS J 2014;281:3816-3827.

46 Dunlop EA, Hunt DK, Acosta-Jaquez HA, Fingar DC, Tee AR: ULK1 inhibits mTORC1 signaling, promotes multisite Raptor phosphorylation and hinders substrate binding. Autophagy 2011;7:737-747.

47 Loffler AS, Alers S, Dieterle AM, Keppeler H, Franz-Wachtel M, Kundu M, Campbell DG, Wesselborg S, Alessi DR, Stork B: Ulk1-mediated phosphorylation of AMPK constitutes a negative regulatory feedback loop. Autophagy 2011;7:696-706. 